\title{
Reverberations from our initial cases of MIS TLIF: Perilous fear of radiation exposure and recollection of our cuts and bruises
}

\author{
Suyash Singh $^{1}$, Jayesh Sardhara ${ }^{2 *}$, Kamlesh Singh Bhaisora ${ }^{3}$, Arun Kumar Srivastava ${ }^{4}$, Sanjay Behari $^{5}$ \\ ${ }^{2,3}$ Assistant Professor, ${ }^{4}$ Associate Professor, ${ }^{5}$ Professor, ${ }^{1-5}$ Dept. of Neurosurgery, ${ }^{1-5}$ Sanjay Gandhi Post Graduate Institute of Medical \\ Sciences, Lucknow, Uttar Pradesh, India
}

*Corresponding Author: Jayesh Sardhara

Email: dr.suyashsingh@gmail.com

\begin{abstract}
Introduction: The increasing demand of MISS TLIF is constraining young spine surgeons to acquire perilous technique. The apprehension for radiation exposure is usually suppressed by surgical satisfaction. The aim of this study was to share our experience of initial cases, occupational hazards faced and patient reported outcome measures (PROM). We intend to highlight the problems encountered in the learning curve and technical pearls we learnt.

Materials and Methods: The study includes initial 30 patients of spondylolisthesis operated by MISS-TLIF. The oswestry disability index (ODI) score and Patient satisfaction score at discharge and 6-months follow up were studied. Postoperative Bridwell's anterior fusion grading used to access fusion rate at 6th month. The occupational risks and job stress was calculated using Staff stress questionnaire and compared to staff members of other sub-speciality. We also noted the number of CARM shots taken in those initial cases.

Results: There was the improvement in the mean ODI score of $61.87 \pm 16.65$ pre operatively to $24.23 \pm 13.85$ in postoperative period; (p< 0.001 ) at six month follow up. Although the median patient's satisfaction score was 3 at discharge, but it improved to 2 at $6^{\text {th }}$ month of follow up. According to Bridwell's fusion grading $62 \%$ of the patients in our study showed Grade 2 fusion, $31 \%$ showed grade 3 fusion. There was significant difference in median stress level of our staff compared other operating room staff members ( $p>0.01$ ). Although the radiation exposure was higher compared to other series, but there was decreasing trend after $10^{\text {th }}$ case.

Conclusion: MISS-TLIF is a safe and efficient technique. After optimal experience and discerning learning curve, the risk of radiation and post operative complications declines. We should advise our operating room staff workers regarding all precautionary steps and assure them regarding the safety of MIS TLIF procedure.
\end{abstract}

Keywords: Minimally invasive spine surgery, TLIF, Computer assisted radio monitoring, Oswestrry disability index, Patients satisfaction score

\section{Key Points}

1. The MISS TLIF technique is demanding with intuitive learning curve and prolonged radiation exposure.

2. The hesitation to acquire new technique, become familiar to instruments and fear of high radiation exposure at initial days, barricades surgeon's inclination to MISS TLIF.

3. There is significant reduction in radiation exposure after 10 cases.

4. Spine surgeons and residents are not at all stressful and enjoy the surgery whereas the staff members not at all feel safe and few $(n=2)$ even wish transfer.

5. There was significant difference in median stress level of our spine O.R. staff compared other staff members.

\section{Introduction}

The era of spinal biomechanics discerns a gradual shift towards minimally invasive spine surgery (MISS). Increasing demand of patients and changing concept of tension band preservation, procreates imperative understanding of MISS. The technique is demanding with intuitive learning curve and prolonged radiation exposure. Among all the anterior or anterio-lateral approaches, Transforaminal lumbar inter-body fusion (TLIF) remains the most popular method of surgical fusion for the treatment of spondylolisthesis. The hesitation to acquire new technique, become familiar to instruments and fear of high radiation exposure at initial days, barricades surgeon's inclination to MISS TLIF. This purpose of our study is to highlight the surgical outcome and patient reported outcome measures in MISS TLIF procedure for spondylolisthesis, in our learning curve focusing the problem of radiation hazards

\section{Materials and Methods}

The article is written according to the Revised Standards for Quality Improvement Reporting Excellence (SQUIRE 2.0). Individual consent from the patient to use clinical and radiological details for publication was taken. Institutional ethical clearance was obtained and there was no conflict of interest or financial support taken.

\section{Patient Spectrum}

For this study, we included our initial 30 patients of spondylolisthesis, surgically managed by MISS TLIF at our institute, from July 2015 to June 2018. All 30 patients of spondylolisthesis were graded radiologically according to the Meyerding's grading system. Demographic, clinical and operative data were obtained through hospital records.

\section{Study Parameters}

For comparisons of pre and postoperative clinical outcome of surgery, we noted the Oswestry disability index (ODI) scoring in the preoperative period and at 6 months follow up period. The surgical outcome was done by postoperative 
lumbar spine X-ray to evaluate degree of screw purchase and degree of fusion using the anterior fusion grade described by Bridwell et al. ${ }^{1,4}$ [Grade I: Fusion with remodelling and trabeculae; Grade II: Graft intact, not fully remodelled, no radiolucency; Grade III: Graft intact, but a definite radiolucency; and, Grade IV: Definitely not fused, collapsed].

Patient satisfaction score was also analysed using a 5-point Likert scale. (Patients were asked: "how satisfied were you with the medical care and back pain problem after surgery?" Response categories included the following: 1 = very satisfied; 2 = somewhat satisfied; 3 = neither satisfied nor dissatisfied; $4=$ somewhat dissatisfied; and $5=$ very dissatisfied). ${ }^{2,3,24}$

The staff members including surgeon, residents, nursing staff and technicians were retrospectively asked Staff stress questionnaire using 5-point Likert scale. (They were asked: "How safe you feel in assisting MIS TLIF cases with CARM radiation?" Response categories included the following: 1 = they feel safe with all precautions; 2 = safe but worried after surgery; $3=$ neither safe nor unsafe, its duty so they don't think on that much; $4=$ unsafe but do not wish to get transfer; and $5=$ very unsafe and wish to get transfer in other surgical wing). Apart from subjective outcomes, we also calculated number of CARM shots used in all 30 cases from stored data.

\section{Exclusion Parameters}

We excluded patients of traumatic spondylolisthesis and patients operated by standard open pedicle screw fixation. We did not operate any case of grade IV spondylolisthesis in our learning curve.

\section{Surgical Procedure}

After marking of mid-spinous line under the fluoroscopy, targeted the lumbar disc space is marked under AP view. Followed by lateral pedicle line is marked by an imaginary line drawn from the lateral border of upper and lower lumbar pedicle in AP view. A $2-3 \mathrm{~cm}$ incision is made for MI-TLIF in lateral pedicle line. Under fluoroscopy guidance first, a guide wire inserted via four pedicles at desired vertebral level (Fig. 1a). The trajectory allows direct corridor to the disc space and both Ipsilateral as well as contralateral foramen. The dilator of smallest diameter is docked over facet joints. The track is further progressively dilated until the largest numbered dilators of $22 \mathrm{~mm}$ sized is docked. The dilator is then replaced by quadrant retractor (MAST retractor) (Fig. 1b). Under microscope inferior articular process of spine along with part of the lamina is drilled. This exposes the underlying foramen. (Fig. 1c,d). We usually use autograft (preserved facet bone) for later interbody fusion TLIF cage. Foraminotomy can be extended by drilling of medial part of superior articular process of lower lumbar vertebrae. The nerve root is retracted medially via nerve hook. Complete transforaminal discectomy is performed. After ensuring complete decompression of bilateral nerve root, banana-shaped or straight single cage filled with autograft inserted in the anterior two third of disc space to maintain lumbar lordosis (Fig. 1e,f). A guide wire is replaced with the percutaneous screw. Rod is placed percutaneously with the same incision.

It is important that the initial skin incision is made with the patients' body habitus in mind (Fig. 1g). ${ }^{3}$ In obese patient skin entry point should be approximately $1 \mathrm{~cm}$ lateral to provide an oblique angle to the pedicle. The Jamshidi needle is then docked over the lateral aspect of the pedicle at " 3 " o clock -position in the eye of the oval pedicle on the anterior/posterior image. The Jamshidi needle is further advanced $20 \mathrm{~mm}$ to $25 \mathrm{~mm}$ so that the needle is beyond the medial border of the pedicle and into the vertebral body. Place a K-wire through the Jamshidi needle followed by the pedicle tap.

Under fluoroscopy four transpedicular guidewire passed at L3/4 level (Fig 1a), then $2.5 \mathrm{~cm}$ incision given lateral to midline for insertion MIA quadrant retractor (Fig.1b), after L3/4 discectomy via transforaminal route bilateral L4 foraminal decompression done form right side (Fig 1c,d). Postoperative CT (Fig.1e,f) lumbar spine reveals restoration of L3/4 foramen height with interbody TLIF cage. The entry point of screw and incision of TLIF closed with subcuticular stitches (Fig.1g). Fig h shows diagrammatic picture of entry point.

\section{Statistical Analysis}

Statistical analysis was performed using SPSS software version 24 (IBM Corp., Armonk, NY, USA). Independent Mann Whitney $U$ test was used to compare the median distribution of study parameters among the cases and controls. A p value of less than 0.05 was considered as significant.

\section{Results \\ Clinical Profile}

The mean age was 42.55 year (range:14-65; 13M:17F). According to the Meyerding's grading system, we operated 17 cases of grade 1 spondylolisthesis and 11 cases of grade 2 spondylolisthesis and 2 case of grade 3 spondylolisthesis. 26 patients had improvement in radiculopathy and 5 had improvement in pre operative motor neurological deficit. 5 had persistent neurological deficit ( 2 motor and 3 sensory). 4 patients had intra operative dural tear (3 managed successfully whereas 1 needed additional acetazolamide and increase in hospital stay duration). 3 patients had instrument related neural injury ( $2 / 3$ had two surgeries). None of the patient needed re-exploration for wound complication including CSF leak. 2 patient had mal-union and 3 patients had wrong site implant insertion which needed removing and reinsertion intra operatively. We also analysed all 30 cases in chronological order and found gradual decrease in complications with a sharp decline after $12^{\text {th }}$ case. 

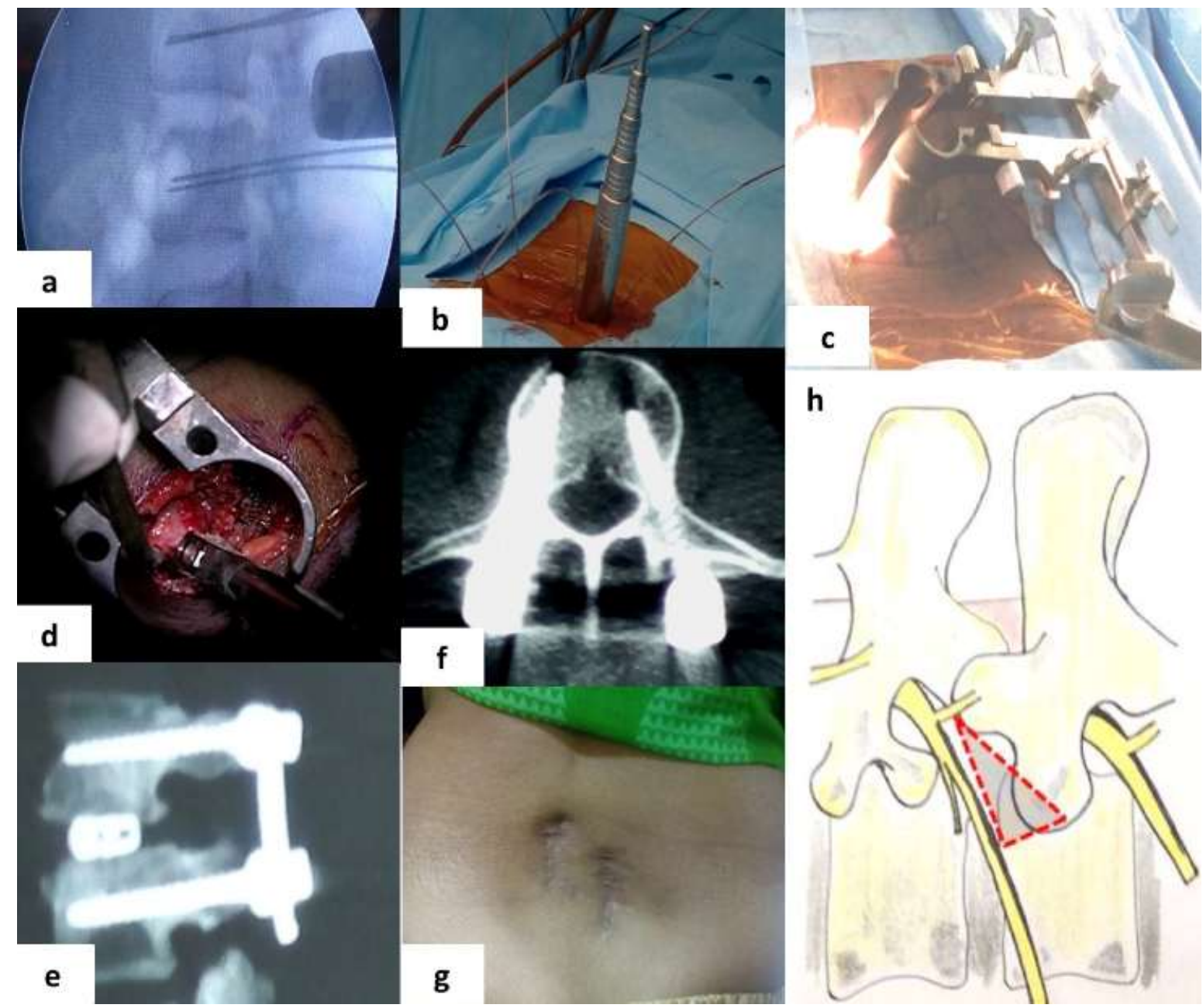

Figure 1: A 46-year male presented with low back ache with neurogenic claudication.X-ray lumbar spine lateral view showing L3/4 grade 1 spondylolisthesis, and MRI suggestive of L3/4 central disc prolapse with canal stenosis. Planned for Minimal invasive transforaminal decompression with transforaminal lumbar interbody fusion (MISS TLIF).

\section{Surgical Outcome}

On radiological follow up according to the Bridwell's fusion grading $62 \%$ of the patients in our study showed Grade 2 fusion, $31 \%$ showed grade 3 fusion at $6^{\text {th }}$ months, and one patient (with grade II spondylolisthesis) presented with TLIF cage subsidence in the spinal canal with limb radiculopathy, so TLIF cage needed removal. Five patient required re-surgery for screw revision. Among them, three patients of MIS TLIF were re-operated due to medial pedicle breach (wrong purchase of pedicle screw) with ipsilateral side limb radiculopathy (severe pain with toe weakness). All the three patients underwent revision of pedicle screw in immediate post operative period when the radiculopathy improved immediately and weakness of toes gradually improved after 2 to 3 weeks. The revision rate was $16.7 \%(5 / 30)$. Mean hospital stay for MI-TLIF was 5.2 days are comparable to other studies. ${ }^{5,6}$

\section{Patient Related Outcome Measures}

The mean pre-operative ODI score was $61.87 \pm 16.65$ which improved to $24.23 \pm 13.85$ at follow-up $(p<0.001)$. Median patient's satisfaction score was 3 at discharge, but it improved to 2 at $6^{\text {th }}$ month of follow up. We calculated independent Staff stress on 5-point questionnaire. We calculated staff stress questionnaire on 5 surgeons, 12 residents, 12 nursing staff and 4 technicians. We further questioned 15 staff member on same questionnaire. The median score among surgeons and residents was 2 , median score among other staff was 4 compared to median score of 1 among staff of other sub-speciality. There was significant difference in median stress level of our spine O.R. staff compared other staff members ( $\mathrm{p}>0.01)$.

We also noted number of CARM shots in our initial surgeries. The median number in initial 10 cases was $48.5 \pm$ 20.5 IQR (28-80), whereas number median in next 20 cases was $18 \pm 7.25$ IQR (12-30). So, there was significant reduction in radiation exposure after 10 cases $(p<0.01)$.

\section{Follow up}

The mean follow-up period in our study was 12.4 months with a range of 7 months to 39 months. 


\section{Discussion}

TLIF was popularized by Harms and Jeszenszky as an alternative to posterior approaches for degenerative spondylolisthesis. ${ }^{1}$ Foley et $a .^{2}{ }^{2}$ described an alternative procedure, the minimally invasive TLIF, with advantage of decrease soft tissue injury but helped to achieve the same desired surgical objective. The advantages of transforaminal route compare to midline approaches are lesser incidence of CSF leak, epidural scarring, postoperative instability and rapid recovery. ${ }^{3}$

The paradigm shift to MISS-TLIF is demanding both in terms of technical challenge and anatomical correlation. There is a world wide trend shifting towards MISS. ${ }^{711}$ Our study results encourage the same shift highlighting certain tricks and tactics to curtail their learning curve. The significant improvement in postoperative ODI score with MISS- TLIF in our initial cases, justifies safe transition from open to MISS.

We believe that initial $10-12$ cases may suffer complications in learning curve. In this article, we intend to highlight that curve is intuitive and complication rates curtail with proper understanding relevant anatomy and familiarity with instruments or image orientation. Studies comparing surgical outcome claim 0-30 cases under learning curve. The larger reviews conclude that the technique is a reliable and effective with significant learning is accomplished after 12 cases and $90 \%$ of the expertise is achieved at around 40 cases. ${ }^{12}$ We believe that the learning curve could be further shortened with adequate experience of open pedicle screw fixation. The revision rate in our study was $16.7 \%$ which is comparable to rates quoted in other larger studies (10\%-20\%). ${ }^{13}$

In spite of high revision rates and resurgery in 5 patients, the median satisfaction at 6th month was good (median PSS=2). At the time of discharge, the patients were somewhat less satisfied (median PSS $=3$ ), which could because of operative site pain or difficulty to ambulate. None of the patient in our study was dissatisfied after surgery. These results may be confounded by educational status of patients and pre operative counselling. The combined improvement in ODI scores and PSS scores justifies that MIS TLIF is safe. We have not compared the scores with patients undergoing open pedicle screw fixation. On reviewing the literature, we found no difference in surgical outcome between MISS TLIF and open TLIF. In the comparison of 345 patients of lumbar spondylolisthesis, significant improvement was found in terms of all functional outcomes in both open Vs MISS groups with no difference in length of hospital stay and 90 days return to work. ${ }^{5}$ The similar result was concluded by Rouben et al with 169 patients of MIS TLIF. ${ }^{6}$ Bing et al studied magnetic resonance imaging of the paraspinal musculature of both the open and MISS patients postoperatively in 187 patients and found significant atrophy of multifidus after open surgery and the muscular architecture was preserved in MISS. He showed that the histopathology of paraspinal muscles which undergone 90 minutes retraction in open surgery had cellular edema and blurring of cell borders contrast to preserved cytoarchitecture in MISS. ${ }^{14}$ Similarly, Wang et al published the result of endoscopic MIS TLIF without GA, suggested that this technique under conscious sedation may represent the feasible alternative to traditional lumbar spine fusion in selected patients. ${ }^{15}$ Parker et al revealed postoperative superficial soft tissue infection was significantly lower in the MIS-TLIF cohorts at $0.6 \%$ vs. $4.0 \%$ in the open-TLIF cohorts in his study. ${ }^{16}$

To best of our knowledge, our study is first to raise concerns of nursing staff and technicians with subjectively highlighting their radiation fear. The radiation hazards under fluoroscopic guidance spine surgery is well known. We found a strange finding that on one hand, surgeons and residents are not at all stressful and enjoy the surgery even knowing the consequences of radiation; whereas, on other hand, the staff members not at all feel safe and few $(n=2)$ even wish transfer. Although there was decreasing trend in number of CARM shots, the mean number of still high $28 \pm$ 16.6SD (median 21). One should be acquainted about the fact that in initial case, radiation exposure and operating time is more. Authors have calculated radiation dosages in cadaveric study and spine models. Rampersaud et al, in his cadaveric study, studied radiation exposure to surgeons and found that average dose rate was $8.3 \mathrm{mrem} / \mathrm{min}, 53.3$ $\mathrm{mrem} / \mathrm{min}$, and $58.2 \mathrm{mrem} / \mathrm{min}$ to neck, torso and hands subsequently. Authors recommend pulsed imaging during fluoroscopy and use of collimation to reduce unnecessary radiation exposure. The hands and face are always at stake under fluoroscopy. Objective assessment of dosimeter could provide better remarks, but its availability and selective use in MISS TLIF again limits dependability. Further prospective studies are needed to establish correlation and measure exact dose hazards. Subsequently, we tried to overcome this limitation in later cases by integrating Neuronavigation system guiding trajectory and pedicle screw fixation.

\section{Technical pearls: Our initial technical experience of MISS TLIF}

1. We preferred bilateral foramen decompression from ipsilateral side, but in the case with severe canal stenosis $(\mathrm{n}=2)$ bilateral decompression and bilateral TLIF insertion is preferable.

2. During facet joint drilling (diamond burr should be used), one should be that ligamentum flavum remains intact till bony decompression is complete; this practice helps us to reduce the incidence of dural tear.

3. We recommend single level L3/4 or L4/5 spondylolisthesis without canal stenosis is an ideal choice for beginners.

4. Grade II or III spondylolisthesis can also be reduced slight modification in using percutaneous distraction method wherein the distractor is fixed over head of percutaneous screw and distraction done under fluoroscopy till complete reduction is achieved. The technique is catalyzed by drilling bilateral facet joint via the different incision between two pedicles on the contralateral side. 
5. Relative contraindication for MIS TLIF: obese patients, more than two level or spondyloptosis (spondylolisthesis grade >II should not be tried in initial 10-12 cases) and scoliotic deformity further complicates the screw fixation and reduction.

\section{Limitations of Study}

The study has relatively short duration of follow-up; hence, the long-term results including fusion rates are unclear. With the small number of patients, it was difficult to conclude about statistical results and comparison. We arbitrarily took initial 30 cases, but it is possible that the effect of learning curve is better reflected in smaller samples. Moreover, the learning curve of MISS also depends on surgical experience of surgeon, patient cooperation and availability of technicians in operative room. The use of electric monitoring may alter the outcome. The choice between open and MISS group depended on patient's consent and surgeon's expertise. Selection bias further decreases power of our study. But our study succeeded in its aim, establishing safety of procedure at even learning period and thereby recommends more and more budding spine surgeons to learn the technique.

\author{
Abbreviations \\ MISS minimally invasive spine surgery \\ TLIF transforminal lumbar Interbody fusion \\ CARM Computer assisted radio monitoring \\ CT Computed tomography \\ $\mathrm{K}$ wire Kirschner wires \\ ODI Oswestrry disability index \\ PSS Patients satisfaction score \\ IQR interquartile range \\ SD standard deviation
}

\section{Conclusion}

MISS-TLIF is a safe and effective procedure in transition learning phase with proper selection of patients. The results are comparable after initial 10-12 cases. The radiation exposure is high in initial 10 cases but declines subsequently. With proper counselling, patient satisfaction is good and fusion rates are acceptable even in short follow up. Overall MISS yields faster recovery due to the advantage of less tissue dissection, decreased blood loss, decreased postoperative pain, shorter hospital stay, and earlier ambulation. We recommend that it is worth walking ahead along the learning curve of MISS.

\section{Conflicts of Interest: None.}

Funding of Source: No funds were received in support of this study.

Acknowledgement: None.

\section{References}

1. Harms JG, Jeszenszky D. "Die posteriore, lumbale, interkorporelle Fusion in unilateraler transforaminaler Technik”. Oper Orthop Traumatol 1998;10(2):90-102.

2. Kambin P. Re: Foley KT, Holly LT, Schwender JD. "Minimally invasive lumbar fusion". Spine (Phila Pa 1976). 2004;29(5):598-9. PubMed PMID: 15129085.

3. Mobbs RJ, Sivabalan P, Li J. "Technique, challenges and indications for percutaneous pedicle screw fixation". J Clin Neurosci 2011;18:741-49. doi: 10.1016/j.jocn.2010.09.019.

4. Bridwell KH, Lenke LG, McEneryKW,. "Anterior structural allografts in the thoracic and lumbar spine". Spine 1995; 20:1410-18.

5. Mummaneni PV, Bisson EF, Kerezoudis P, "Minimally invasive versus open fusion for Grade I degenerative lumbar spondylolisthesis: analysis of the Quality Outcomes Database." Neurosurg Focus 2017;43(2):E11. doi: 10.3171/2017.5.FOCUS17188.

6. Rouben D, Casnellie M, Ferguson M. "Long-term durability of minimal invasive posterior transforaminal lumbar interbody fusion: a clinical and radiographic follow-up". J Spinal Disord Tech 2011;24: 288-96.

7. Kanter AS, Mummaneni PV. "Minimally invasive spine surgery”. Neurosurg Focus 2008;25:E1. doi: 10.3171/FOC/2008/25/8/E1.

8. Kan P, Schmidt MH. "Minimally invasive thoracoscopic approach for anterior decompression and stabilization of metastatic spine disease". Neurosurg Focus 2008;25(2):E8. doi: 10.3171/FOC/2008/25/8/E8.

9. Assaker R. "Minimal access spinal technologies: state-of-theart, indications, and techniques". Joint Bone Spine 2004; 71:459-69.

10. Selznick LA, Shamji MF, Isaacs RE. "Minimally invasive interbody fusion for revision lumbar surgery: technical feasibility and safety". J Spinal Disord Tech 2009;22:207-13.

11. Kerr SM, Tannoury C, White AP. "The role of minimally invasive surgery in the lumbar spine". Oper Tech Orthop 2007;17:183-9.

12. Silva PS, Pereira P, Monteiro P, "Learning curve and complications of minimally invasive transforaminal lumbar interbody fusion". Neurosurg Focus 2013;35(2):E7. doi: 10.3171/2013.5.FOCUS13157.

13. Sclafani JA, Kim CW. "Complications associated with the initial learning curve of minimally invasive spine surgery: A systematic review". Clin Orthop Relat Res 2014; 472:1711-7.

14. Li YB, Wang XD, Yan HW. "The Long-term Clinical Effect of Minimal-Invasive TLIF Technique in 1-Segment Lumbar Disease”. Clin Spine Surg 2017;30:E713-E719. doi: 10.1097/BSD.0000000000000334.

15. Michael Y. Wang, MD, Jay Grossman, MD. “Endoscopic minimally invasive transforaminal interbody fusion without general anesthesia: initial clinical experience with 1-year follow-up". Neurosurgical focus 2016; 40: E13

16. Parker SL, Adogwa O, Witham TF, "Post-operative infection after minimally invasive versus open transforaminal lumbar interbody fusion (TLIF): literature review and cost analysis". Minim Invasive Neurosurg 2011;54:33-7. doi: 10.1055/s-00301269904.

17. Yan DL, Li J, Gao LB, "Comparative study on two different methods of lumbar interbody fusion with pedicle screw fixation for the treatment of spondylolisthesis". Zhonghua Wai 
Ke Za Zhi 2008;46(7):497-500. Chinese. PubMed PMID: 18785557.

18. Zhuo X, Hu J, Li B, "Comparative study of treating recurrent lumbar disc protrusion by three different surgical procedures". Zhongguo Xiu Fu Chong Jian Wai Ke Za Zhi 2009 Dec;23(12):1422-6. Chinese. PubMed PMID: 20073301.

19. Humphreys SC, Hodges SD, Patwardhan AG. "Comparison of posterior and transforaminal approaches to lumbar interbody fusion”. Spine (Phila Pa 1976). 2001;26(5):567-71. PubMed PMID: 11242386.

20. Zhang Q, Yuan Z, Zhou M, et al. "A comparison of posterior lumbar interbody fusion and transforaminal lumbar interbody fusion: a literature review and meta-analysis". $B M C$

Musculoskelet Disord 2014;15:367. doi:10.1186/1471-247415-367. Review. PubMed PMID: 25373605; PubMed Central PMCID: PMC4232693.

21. Mobbs RJ, Sivabalan P, Li J, et al. "Hybrid technique for posterior lumbar interbody fusion: a combination of open decompression and percutaneous pedicle screw fixation". Orthop Surg 2013;5(2):135-41. doi: 10.1111/os.12042.PubMed PMID: 23658050.

22. Phan K, Mobbs RJ, Rao PJ. "Foraminal height measurement techniques. J Spine Surg 2015;1(1):35-43. doi:10.3978/j.issn.2414-469X.2015.07.01.

23. Mobbs RJ, Phan K, Malham G, "Lumbar interbody fusion: techniques, indications and comparison of interbody fusion options including PLIF, TLIF, MI-TLIF, OLIF/ATP, LLIF and ALIF”. J Spine Surg 2015;1(1):2-18. doi:10.3978/j.issn.2414469X.2015.10.05.
24. Dhall SS, Wang MY, Mummaneni PV. "Clinical and radiographic comparison of mini-open transforaminal lumbar interbody fusion with open transforaminal lumbar interbody fusion in 42 patients with long-term follow-up: clinical article”. J Neurosurg Spine 2008; 9:560-565.

25. Par kY, Ha JW. "Comparison of one-level posterior lumbar interbody fusion performed with a minimally invasive approach or a traditional open approach". Spine (Phila Pa 1976). 2007;32:537-43.

26. Singh S, Sardhara JC, Khatri D, Joseph J, Parab AN, Bhaisora $\mathrm{KS}$, “Technical pearls and surgical outcome of early transitional period experience in minimally invasive lumbar discectomy: A prospective study". J Craniovertebr Junction Spine 2018;9(2):122-29. doi: 10.4103/jcvjs.JCVJS_47_18.

How to cite this article: Singh S, Sardhara J, Bhaisora KS, Srivastava AK, Behari S. Reverberations from our initial cases of MIS TLIF: Perilous fear of radiation exposure and recollection of our cuts and bruises. India J Neurosci 2019;5(2):81-6. 